\title{
Evaluación del tratamiento con infliximab en enfermos con artritis inflamatoria refractaria a drogas habituales
}

\author{
Cristián Labarca S, Loreto Massardo V, \\ Paula I G arcía $\mathbf{M}^{1}$, Sergio Jacobelli G. \\ Effectiveness of infliximab in patients \\ with inflammatory arthritis refractory \\ to conventional treatment
}

Background: Tumor necrosis factor antagonists are useful in the treatment of several chronic inflammatory immune mediated diseases. Aim: To assess the effects of infliximab in 21 patients with inflammatory arthropaties, refractary to conventional treatment. Patients and methods: Eleven patients with rheumatoid arthritis, seven with psoriatic arthritis and three with spondyloarthritis were treated. The mean duration of the diseases was 10 years. Infliximab was administered intravenously in a dose of $3 \mathrm{mg} / \mathrm{kg}$ body weight. A median of 6 doses in 8 months was administered. Effectiveness was assessed in 19 patients that received three or more doses. Results: Infliximab was effective in 16 patients (10 with rheumatoid arthritis, four with psoriasis and two with spondyloarthritis) and ineffective in three. In responsive patients, a reduction in the number of inflammed joints and morning stiffness and an improvement in functional capacity was observed. Fifteen of the 16 patients perceived an improvement in their health status. This answer was concordant with concomitant medical evaluation in 15. Patients that maintained the treatment felt very well, well or regular, whereas five of six patients that discontinued the treatment felt ill. Thirteen patients had adverse effects. Treatment was discontinued in two patients due to drug induced lupus, allergy in 2, hypertension in one, high costs in three and lack of response in three. Conclusions: Infliximab reduced arthritic activity in 16 of 19 patients with severe treatment refractary arthritis (Rev Méd Chile 2003; 131: 1157-64).

(Key Words: Arthritis; Infliximab; Tumor necrosis factor)

Recibido el 29 de enero, 2003. Aceptado en versión corregida el 4 de agosto, 2003.

Departamento de Inmunología Clínica y Reumatología. Facultad de Medicina, Pontificia Universidad Católica de Chile.

${ }^{1}$ Enfermera Universitaria

Correspondencia a: Dr. Cristián Labarca Solar. Departamento de Inmunología Clínica y Reumatología. Facultad de Medicina, Pontificia Universidad Católica de Chile. Marcoleta 352, Santiago - Chile. Teléfono 6397750. Fax 6324937. E mail: clabarcasolar@hotmail.com 
L a artritis reumatoidea (AR) es una enfermedad con una expresión clínica variable ${ }^{1}$. Un porcentaje de los enfermos tiene una evolución relativamente benigna y otro desarrolla gran destrucción articular, deformaciones, incapacidad funcional marcada y presentan una mortalidad aumentada comparada con la de la población general. Las terapias con drogas antirreumáticas modificadoras de la AR (DMARDs) pueden inducir remisión, reducir la gravedad del curso de la enfermedad, el grado de invalidez y la mortalidad, especialmente si éstas son utilizadas al inicio. Sin embargo, hay un grupo de pacientes que no responde a estos medicamentos. En ellos se ha propuesto con éxito el uso de antagonistas del factor de necrosis tumoral (TNF- $\alpha)^{2}$.

Las citoquinas juegan un papel central en la patogenia de las enfermedades inflamatorias crónicas como la $\mathrm{AR}$, artritis psoriática y espóndilo artropatías (EAA). El TNF- $\alpha$ derivado de macrófagos es una citoquina responsable de la regulación de las interacciones entre las células del sistema inmune como fibroblastos y células endoteliales. Se expresa en cantidades aumentadas en el suero y líquido sinovial de pacientes con AR y promueve la liberación de otras citoquinas inflamatorias como la interleuquina IL-1, IL-6 e IL-8 y estimula la liberación de proteasas. La importancia clave del TNF- $\alpha$ en la patogénesis de la AR ha sido demostrada porque su bloqueo resulta en una mejoría substancial de la enfermedad ${ }^{3}$.

Clínicamente se han diseñado dos modos de bloquear el TNF- $\alpha$ : uno es mediante el uso de su receptor soluble, el etanercept (Enbrel ${ }^{\circledR}$ ), lo que evita que el TNF- $\alpha$ se una al receptor de membrana celular. Otro es la utilización de anticuerpos monoclonales contra TNF- $\alpha$. Estos pueden ser quiméricos (humano $75 \%$ y murino $25 \%$ ) como el infliximab (Remicade ${ }^{\circledR}$ ) o humanizados como el adalimumab (Humira ${ }^{\circledR}$ ). La eficacia del infliximab es similar a la del etanercept. Aproximadamente $70 \%$ de los enfermos con AR experimenta una disminución importante de la actividad inflamatoria entre 8 a 12 semanas de uso ${ }^{3}$.

En la última década la introducción de las terapias biológicas con anticuerpos anti-TNF- $\alpha$ causó una mejoría muy importante de la calidad de vida de pacientes con enfermedad de $\mathrm{Crohn}^{4}$, $\mathrm{AR}^{5}$, psoriasis ${ }^{6}$ y $\mathrm{EAA}^{7}$ que eran refractarios al tratamiento con drogas inmunosupresoras con- vencionales. El uso de estos agentes biológicos ha demostrado una disminución del número de articulaciones inflamadas, del dolor y mejoría de la capacidad funcional en AR. Según los criterios de repuesta a tratamiento del American College of Rheumatology (ACR-20) ${ }^{8}$, los estudios de eficacia de infliximab combinado con metotrexate, analizados a las 54 semanas, muestran una mejoría que fluctúa entre $42 \%$ y $59 \%$ de los pacientes con AR refractaria a metotrexate. Además se retrasa la aparición de erosiones radiológicas y la disminución del espacio articular en $\mathrm{AR}^{3,5,9}$.

El objetivo de este estudio fue evaluar nuestra experiencia clínica con el uso de infliximab obtenida en 21 pacientes con artropatías inflamatorias refractarias a tratamiento con DMARDs.

\section{PACIENTES Y MÉTODO}

En el Departamento de Reumatología del Hospital Clínico de la Pontificia Universidad Católica de Chile, iniciaron terapia con infliximab 21 pacientes con artritis activa entre mayo de 2000 y agosto de 2002. Doce eran mujeres y 9 hombres. La mediana de edad al inicio de la terapia fue 40 años (intervalo 29-63). La mediana de la duración de la artritis fue 10 años (1-30). En todos los casos el tratamiento habitual de la artritis inflamatoria había fracasado. Habían utilizado entre 1 y 6 DMARDs (mediana 3): metotrexate, leflunomida, azulfidine, salazopirina, azatioprina, cloroquina, hidroxicloroquina, penicilamina, sales de oro, ciclosporina A o ciclofosfamida, en diferentes combinaciones.

Once pacientes cumplían los criterios de diagnóstico para artritis reumatoidea del ACR de 198710, 9 tenían factor reumatoideo positivo. Siete tenían artritis psoriática. Tres cumplían los criterios europeos para espondiloartritis anquilosante (EAA) ${ }^{11}$. Los enfermos con AR tenían una mediana de 19 articulaciones inflamadas (de 8 a 22) y la rigidez matutina era de 60 min (de 15 a 300). Los siete con artritis por psoriasis tenían artritis en una mediana de 2 articulaciones (2-5). Dos con EAA tenían artritis en 8 y 5 articulaciones y el otro un lumbago inflamatorio con sacro-ilitis rebelde a tratamiento.

Infliximab. Remicade ${ }^{\circledR}$ fue donado gentilmente por Schering-Plough para 1 año de tratamiento de 12 enfermos. El medicamento se administró en 
hospitalización transitoria por infusión intravenosa (iv) en 2 a 3 ampollas de $100 \mathrm{mg}$ por vial a las semanas 0,2 y 6 y luego cada 8 ó 12 semanas 0 más. La mediana de la dosis utilizada fue $3,1 \mathrm{mg} /$ $\mathrm{kg}$ de peso (1,4-4,4). La mediana del número de dosis fue 6 (1-15) y la mediana de la dosis acumulada fue $1.000 \mathrm{mg}$ (100-5.000). La duración del tratamiento tuvo mediana de 8 meses (1-28). Todos los pacientes con AR o EAA utilizaron combinación con metotrexate, azatioprina o leflunomida. Dos enfermos con artritis por psoriasis no utilizaron la combinación con DMARD.

La respuesta a terapia con Remicade ${ }^{\circledR}$ se calificó como eficaz de acuerdo con un protocolo establecido que consideraba los criterios siguientes: menor duración de la rigidez matutina, disminución del número de articulaciones inflamadas, disminución del puntaje en el cuestionario Health Assessment Questionnaire (HAQ) (puntaje 0-3) ${ }^{12}$ y calificación por el médico tratante como mejoría de la artitis en comparación con el ingreso. Los pacientes con AR se evaluaron según los criterios de repuesta a tratamiento ACR- $20^{8}$. En consideración a que la respuesta a infliximab es rápida, observable en un plazo que va de pocos días a 4 meses $^{13}$, para el análisis de respuesta a terapia evaluamos a los 19 enfermos que recibieron 3 o más dosis. Las razones de suspensión de tratamiento y efectos adversos se consignaron mediante revisión de las fichas clínicas en los 21 pacientes.

Recaída. Se evaluó en aquellos enfermos que, habiendo obtenido una respuesta favorable, suspendieron el infliximab. Se consideró que presentaban una recaída de la artritis de acuerdo con la opinión del médico tratante y por revisión de los datos clínicos.

Seguimiento. Los pacientes fueron observados desde la primera dosis de infliximab a octubre de 2002, por una mediana de 24 meses (de 3 a 32 meses). La mediana del lapso entre la última dosis recibida y el fin del estudio fue de 11 meses (de 1 a 28).

Cuestionario telefónico. Se diseñó una entrevista telefónica para conocer la percepción de los enfermos del estado de salud a octubre de 2002. En ella se consultó la valoración del enfermo sobre la eficacia del infliximab. La entrevista telefónica estructurada es una metodología de evaluación e intervención en pacientes con enfer- medades crónicas articulares ${ }^{14}$. Fue aplicada por una enfermera entrenada en reumatología. Consistió en dos preguntas con 5 opciones de respuesta cada una, originadas de la encuesta Medical Outcomes Study Short Form-Health Survey 36 (MOS-SF-36) que consta de 20 ítemes ${ }^{15}$.

1. Comparado con cuando comenzó el tratamiento con infliximab. ¿Cómo se encontraba su salud cuando recibió la última dosis?

Mucho mejor. Algo mejor. Igual.

Algo peor. Mucho peor.

2. Ud. considera que su salud hoy es:

Excelente. Muy buena. Buena. Regular. Mala.

Dieciséis (76\%) pacientes contestaron la encuesta telefónica. Nueve continuaban en tratamiento y 7 lo habían suspendido. Se comprobó la concordancia entre la entrevista telefónica y el examen clínico en 5 enfermos que continuaban en tratamiento y en 3 que lo habían suspendido. Cuatro de los 5 enfermos que no fueron ubicados para la entrevista telefónica habían suspendido el medicamento.

Estadística. En este estudio descriptivo los datos se muestran en mediana con su intervalo o porcentaje. En los pacientes con AR comparamos las medianas del HAQ, del número de articulaciones inflamadas y de la rigidez matutina al iniciar el tratamiento con infliximab y al finalizar éste mediante la prueba no paramétrica de Wilcoxon.

Consentimiento informado. Al inicio del estudio cada paciente firmó un consentimiento informado en el cual se explicó a los pacientes los beneficios y probables efectos adversos del tratamiento, así como su condición al terminar la donación del laboratorio farmacéutico. Este trabajo contó con la aprobación del Comité de Ética del Centro de Investigaciones Médicas de la Facultad de Medicina.

\section{RESULTADOS}

Eficacia. Obtuvieron una buena respuesta 16 (84\%) de 19 pacientes tratados con 3 o más dosis de infliximab. Diez de once con AR, 4 de seis con psoriasis y 2 de tres con EAA. En la mayoría de los casos la mejoría se observó luego de la $2^{\mathrm{a}}$ dosis. En los 10 enfermos con AR la mediana del número de articulaciones inflamadas disminuyó de 19 a 4 
(prueba de Wilcoxon valor $\mathrm{p}=\mathrm{NS}$ ), el HAQ disminuyó de 1,3 a 0,6 puntos ( $\mathrm{p}=\mathrm{NS}$ ) y la duración de la rigidez matutina de 60 a $25 \mathrm{~min}(\mathrm{p}=0,024)$. Con una mediana de 8 meses de duración del tratamiento, los 10 pacientes cumplieron con los criterios de respuesta a tratamiento ACR-20.

Un enfermo con artritis psoriática presentó una mejoría impresionante de la artritis y de la piel, y tres presentaron una marcada mejoría de la artritis. Dos enfermos con EAA tuvieron una mejoría notable de la artritis periférica desde la primera infusión iv.

En dos casos (AR: 1 y artritis psoriática: 1) no se produjo mejoría de la artritis luego de haber recibido 3 dosis de infliximab, por lo que suspendieron el tratamiento. Un tercer paciente con EAA tuvo una mejoría notable con las primeras dos dosis, pero luego sus molestias se exacerbaron a los pocos días de administrada la droga. A pesar de esto, continuó hasta un total de 9 dosis y se consideró el infliximab como no eficaz.

No se consideraron en el análisis de eficacia dos pacientes con artritis psoriática: uno no quiso continuar luego de la segunda dosis por no presentar respuesta y el otro paciente recibió sólo una dosis por crisis hipertensiva durante la infusión iv.

Analizados en octubre de 2002, de los 16 pacientes en que el infliximab fue eficaz, 9 continuaban en tratamiento con una mediana de cinco dosis (3-15). De éstos, 4 nunca tuvieron efectos adversos y en los otros 5 éstos fueron transitorios. Los siete restantes habían suspendido,
4 por efecto adverso entre la $3^{\underline{a}}$ y la $7 \underline{a}$ dosis y 3 lo habían suspendido entre la $3^{\underline{a}}$ y $8^{\underline{a}}$ dosis por el costo alto del medicamento. Los 7 presentaron una recaída de la artritis.

Opinión del enfermo. Con el objeto de conocer la opinión de los enfermos sobre el efecto del tratamiento a la última dosis, y su percepción de salud, se hizo una encuesta telefónica que fue respondida por $16(76 \%)$ de los pacientes de la serie: nueve que continuaban terapia y 7 que la habían suspendido. En comparación con el inicio, el estado de salud al final del tratamiento fue mucho mejor en 10, algo mejor en 5 e igual en 1. En la Figura 1 se muestra la respuesta a la pregunta 1 según diagnóstico.

Desde un punto de vista clínico, en 14 casos el infliximab fue eficaz y en 2 no. En 15 de los 16 enfermos hubo concordancia con la opinión del paciente. Sólo en uno que se consideró algo mejor, el tratamiento fue calificado como no eficaz por el médico.

En relación con la pregunta: «Cómo considera su salud hoy?» efectuada en octubre de 2002, cuatro de 9 pacientes que seguían con infliximab contestaron que la salud era buena o excelente y 5 que era regular. Entre los 7 que no siguieron con infliximab, en 5 la salud fue calificada como mala. Los 7 pacientes habían recibido la última dosis de infliximab hacía un lapso que tuvo una mediana de 18 meses (entre 3 y 23 meses atrás). Tabla 1.

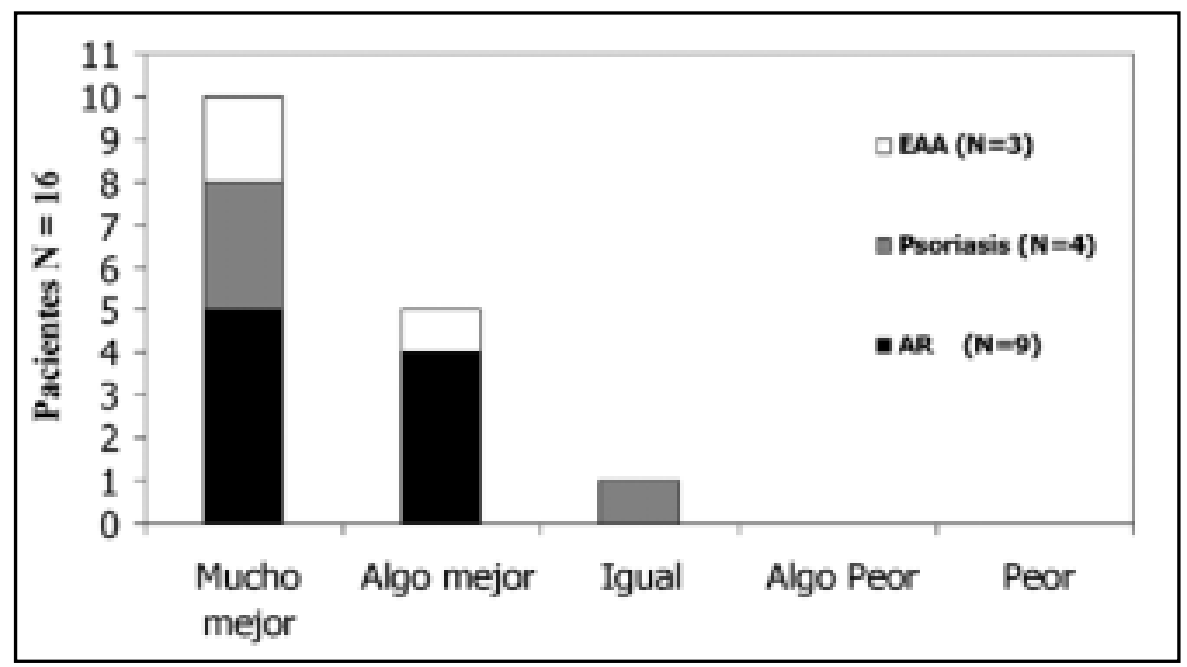

FIguRa 1. Comparado con cuándo comenzó el tratamiento con Infliximab. ¿Cómo se encontraba su salud cuando recibió la última dosis? Respuesta de 16 pacientes según diagnóstico. 
Efectos adversos. Trece pacientes (62\%) de los 21 tratados tuvieron algún efecto adverso: alergia a la infusión iv: 7, hipertensión arterial durante la infusión: 2, lupus inducido por drogas: 2 (exantema cutáneo, anticuerpos antinucleares positivos y anti-dsDNA por IFI en Crithidia luciliae; el cuadro se resolvió con la suspensión de la droga. En un caso se usó prednisona $20 \mathrm{mg}$ al día por 1 mes). Otro enfermo tuvo positividad para anticuerpos antinucleares y tromboflebitis en el sitio de punción. Un enfermo tuvo reactivación de un síndrome de Guillain-Barré. Después de mejorado de esta complicación, se reinició el infliximab a pedido del paciente, en dosis bajas, sin inconvenientes. No hubo infecciones en la serie.

Suspensión. Suspendieron el tratamiento 11 enfermos (53\%). Cinco por efectos adversos (2 por lupus inducido por drogas, 2 alergia a la infusión iv, 1 hipertensión en la primera infusión iv), 3 por falta de respuesta y 3 por el costo elevado del medicamento (Tabla 2).

\section{Tabla 1. Percepción de salud de 16 pacientes con artritis refractaria tratados con infliximab}

\begin{tabular}{|c|c|c|}
\hline \multirow[b]{2}{*}{$\begin{array}{l}\text { ¿Cómo considera } \\
\text { su salud hoy? }\end{array}$} & \multicolumn{2}{|c|}{ ratamiento con Infliximab } \\
\hline & $\begin{array}{l}\text { Continúan } \\
(\mathrm{n}=9)\end{array}$ & $\begin{array}{l}\text { Suspenden } \\
\quad(\mathrm{n}=7)\end{array}$ \\
\hline Excelente & 3 & 0 \\
\hline Muy buena & 0 & 0 \\
\hline Buena & 1 & 1 \\
\hline Regular & 5 & 1 \\
\hline Mala & 0 & 5 \\
\hline $\begin{array}{l}\text { Meses desde la última dosis de } \\
\text { infliximab; mediana (intervalo) }\end{array}$ & $3(1-8)$ & $18(3-23)$ \\
\hline
\end{tabular}

\section{Tabla 2. Características de los pacientes, respuesta al tratamiento y efectos adversos} en 21 pacientes tratados con infliximab

\begin{tabular}{|c|c|c|c|c|c|c|c|c|c|}
\hline Diagnóstico & $\begin{array}{l}\text { Edad, } \\
\text { (años) }\end{array}$ & Sexo & $\begin{array}{l}\text { Duración } \\
\text { artritis, } \\
\text { (años) }\end{array}$ & $\begin{array}{l}\text { DMARDs } \\
\text { utilizados } \\
\text { (n) }\end{array}$ & $\begin{array}{l}\text { Dosis } \\
\text { infliximab } \\
\text { (n) }\end{array}$ & $\begin{array}{c}\text { Respuesta a } \\
\text { Infliximab }\end{array}$ & $\begin{array}{c}\text { Terapia a } \\
\text { octubre } 2002\end{array}$ & $\begin{array}{l}\text { Causa de la } \\
\text { suspensión }\end{array}$ & $\begin{array}{l}\text { Efectos } \\
\text { adversos }\end{array}$ \\
\hline AR & 29 & F & 13 & 4 & 5 & Sí & Continúa & Continúa & $\operatorname{Sin}$ \\
\hline AR & 33 & F & 2 & 2 & 15 & Sí & Continúa & Continúa & Alergia \\
\hline AR & 35 & F & 2 & 4 & 5 & Sí & Continúa & Continúa & Anticuerpo antinuclear y flebitis \\
\hline AR & 32 & F & 20 & 3 & 11 & Sí & Continúa & Continúa & Sin \\
\hline AR & 49 & F & 30 & 3 & 8 & Sí & Suspendida & Costo & Sin \\
\hline AR & 57 & F & 4 & 3 & 6 & Sí & Suspendida & Adverso & Lupus inducido por drogas \\
\hline $\mathrm{AR}$ & 58 & $\mathrm{~F}$ & 7 & 2 & 7 & Sí & Suspendida & Adverso & Lupus inducido por drogas \\
\hline$A R$ & 45 & $\mathrm{~F}$ & 19 & 3 & 3 & Sí & Suspendida & Adverso & Alergia \\
\hline $\mathrm{AR}$ & 52 & $\mathrm{M}$ & 3 & 2 & 8 & Sí & Suspendida & Costo & $\operatorname{Sin}$ \\
\hline $\mathrm{AR}$ & 32 & F & 8 & 3 & 6 & Sí & Suspendida & Adverso & Alergia \\
\hline $\mathrm{AR}$ & 33 & $\mathrm{~F}$ & 10 & 3 & 3 & No & Suspendida & Adverso & Alergia \\
\hline EAA & 50 & M & 20 & 2 & 5 & Sí & Continúa & Continúa & $\operatorname{Sin}$ \\
\hline EAA & 40 & $\mathrm{~F}$ & 17 & 6 & 3 & Sí & Continúa & Continúa & $\operatorname{Sin}$ \\
\hline EAA & 33 & M & 9 & 3 & 9 & No & Continúa & Continúa & Alergia \\
\hline Psoriasis & 33 & M & 10 & 3 & 5 & $\mathrm{~S}^{\prime}$ & Continúa & Continúa & Síndrome de Guillain-Barré \\
\hline Psoriasis & 55 & $\mathrm{~F}$ & 15 & 3 & 5 & Sí & Continúa & Continúa & Alergia \\
\hline Psoriasis & 36 & M & 1 & 2 & 5 & $\mathrm{~S}_{1}^{\prime}$ & Continúa & Continúa & Alergia \\
\hline Psoriasis & 37 & M & 8 & 1 & 3 & Sí & Suspendida & Costo & $\operatorname{Sin}$ \\
\hline Psoriasis & 40 & M & 10 & 3 & 3 & No & Suspendida & Ineficaz & Hipertensión arterial \\
\hline Psoriasis & 63 & M & 26 & 3 & 1 & ND & Suspendida & Adverso & Hipertensión arterial \\
\hline Psoriasis & 49 & M & 12 & 2 & 2 & ND & Suspendida & No continúa & Sin \\
\hline
\end{tabular}

AR: artritis reumatoidea; EAA: Espondiloartritis anquilosante; ND: eficacia no determinada. 


\section{DisCUSIÓN}

El uso de antagonistas TNF- $\alpha$ estableció un nuevo estándar en el tratamiento de las artropatías inflamatorias. Los antagonistas del TNF- $\alpha$ poseen un potente efecto antiinflamatorio al ser usados en pacientes con AR precoz o establecida y se ha postulado que las terapias biológicas debieran iniciarse precozmente si el paciente no responde rápido a las terapias habituales, de modo de evitar la destrucción articular radiológica y el deterioro de la calidad de vida que ocurre en pacientes con artritis $^{3}$

El infliximab fue muy efectivo para el tratamiento de la artritis en 16 de 19 enfermos graves con artritis inflamatorias devastadoras, algunos postrados en su cama, y que no habían respondido de 1 a 7 drogas inmunosupresoras. La eficacia clínica se mantuvo durante el período de tratamiento, que fue relativamente corto: 8 meses. $\mathrm{Si}$ se considera que estos enfermos tenían una AR refractaria al tratamiento habitual, el uso de este agente biológico mejoró significativamente el pronóstico de la enfermedad, lo que incluyó reanudar el trabajo en oficina o reasumir las labores de la casa en los enfermos que respondieron. Durante el primer año de uso las causas de la suspensión del tratamiento en 11 enfermos (53\%) fueron los efectos adversos en 5 casos, falta de eficacia en $3 \mathrm{y}$ el alto costo en 3 . Todos los pacientes recayeron después de la suspensión.

De los 9 enfermos que continuaban con infliximab y que contestaron la encuesta telefónica, 4 calificaron su salud como excelente o buena y 5 una salud regular a pesar de estar mejor o mucho mejor comparativamente con el inicio del infliximab. Los enfermos tenían una mediana de 10 años de artritis grave, con un daño acumulado de cierta magnitud, lo que probablemente influyó en la percepción de salud de los enfermos.

Este estudio sugiere que el uso de una encuesta telefónica en reumatología puede ser de utilidad en situaciones en que es necesario el seguimiento de enfermos que tienen dificultad para acudir al hospital. El $76 \%$ de la serie respondió la encuesta. La eficacia del medicamento según el médico y el paciente concordó en 15 casos. Las preguntas de la encuesta forman parte de la evaluación de los cuestionarios de impacto de salud MOS-SF 36 que miden el estado de salud en varios aspectos que evalúan áreas que no se relacionan solamente con la parte física, sino con otros conceptos y percepciones de salud ${ }^{15}$.

Las alergias e infecciones son complicaciones comunes para todos los antagonistas del TNF- $\alpha^{3}$. Las reacciones en relación con la infusión se observan durante 1 a 2 h después, ocurren hasta en $22 \%$ de los pacientes tratados, a veces se acompañan de fiebre, escalofríos o manifestaciones cardio-pulmonares entre las que se encuentran hipertensión arterial, hipotensión, disnea 0 dolor toráxico. En menos de 1\% hay prurito o urticaria. Manifestaciones serias como choque anafiláctico o convulsiones se han descrito en menos de 1\% de los pacientes. En nuestra serie la alergia a la infusión fue la complicación más frecuente, 33\% (7 pacientes) pero sólo 14\% (3 pacientes) suspendió la terapia por este motivo. Un 10\% (2 pacientes) tuvo hipertensión arterial en la infusión. Es recomendable que el medicamento sea aplicado por personal entrenado especialmente. El uso de infliximab en pacientes con insuficiencia cardíaca está contraindicado. En Europa y EE.UU. muchos enfermos desarrollaron reactivación de la tuberculosis (TBC) o TBC en sitios atípicos durante el tratamiento ${ }^{16}$. En nuestra serie no hubo infecciones. La recomendación actual es obtener una radiografía de tórax normal antes de iniciar tratamiento y asociar profilaxis si hay TBC antigua.

Mohan y $\operatorname{col}^{17}$ describieron enfermedades neurológicas con desmielinización durante terapia con anti-TNF- $\alpha$. En nuestra serie un enfermo con artritis devastadora y extensa psoriasis cutánea, presentó una recaída de un síndrome de GuillianBarré luego de 16 años, al mes de la segunda dosis de infliximab. Como recayera de la artritis 1 mes después de la suspensión del infliximab, solicitó reanudar el tratamiento con excelente respuesta y sin otros problemas ${ }^{18}$.

Se sabe que este tratamiento puede inducir anticuerpos antiinfliximab, lo que disminuiría su eficacia. Por esto se aconseja su uso con algún inmunosupresor ${ }^{3,19}$. Algunas de las complicaciones observadas con el uso del infliximab son el desarrollo de anticuerpos antinucleares (AAN) y el lupus inducido por drogas. Tres pacientes con AR (dos seronegativas y una seropositiva) desarrollaron AAN durante el tratamiento; dos hicieron un 
lupus inducido por drogas, que cumplieron 3 criterios para el diagnóstico de esta afección con sólo compromiso cutáneo evanescente, AAN y antiDNA positivos y que cedió al suspender el infliximab, pero la artritis recayó 20 . No se ha demostrado claramente que el uso de antagonistas TNF- $\alpha$ se relacionen con el desarrollo de linfomas ${ }^{21}$.

Este trabajo comunica una experiencia nacional de corta duración con uso de un anti-TNF- $\alpha$, llevada a cabo en 21 enfermos con diversas artropatías inflamatorias. El 84\% (16/19) tuvo una mejoría de la artritis que fue temporal mientras la droga estaba en uso. Los 10 (91\%) enfermos con AR que cumplieron con los criterios de respuesta ACR-20 refirieron un cambio notable en su estado de salud, a pesar de la morbilidad del medicamento. El $43 \%$ (7/16) debió suspender: 3 por costo elevado y 4 por efectos adversos en el primer año de seguimiento. No hubo una mortalidad asociada con el uso del medicamento. En conclusión el infliximab, al igual que en otros estudios, fue eficaz en nuestro pequeño grupo de pacientes con artritis inflamatoria grave.

Las drogas anti-TNF- $\alpha$ así como los DMARDs sólo son eficaces mientras se mantiene su uso, sin embargo un gran número de enfermos abandona estos medicamentos por diversas razones; no se

\section{REFERENCIAS}

1. Massardo L, Aguirre V, García ME, Cervila V, Nicovani S, GonZÁlez A ET al. Clinical Expression of Rheumatoid Arthritis in Chilean Patients. Semin Arthritis Rheum 1995; 25: 203-13.

2. MatTeSOn EL. Rheumatoid arthritis. Treatment. In: Klippel J, editor. Primer on the Rheumatic Diseases. Atlanta: Arthritis Foundation, 2001; 225-31.

3. Keystone E. Tumor necrosis factor-alfa blockade in the treatment of rheumatoid arthritis. Rheum Dis Clin NA 2001; 27: 427-43.

4. Targan SR, Hanauer SB, van Deventer SJ, Mayer L, Present DH, Braakman T et al A short-term study of chimeric monoclonal antibody cA2 to tumor necrosis factor alpha for Crohn's disease. Crohn's Disease cA2 Study Group. N Engl J Med 1997; 337: 1029-35.

5. Main R, Breedveid F, Kalden J, Somien J, Davis D et AL. Randomized phase III trial of infliximab (chimeric anti-TNF- $\alpha$ monoclonal antibody) ver- debe olvidar que el metotrexate es el DMARD que se mantiene en un mayor porcentaje de enfermos con AR, lo que alcanza a $50 \%$ de los pacientes a 5 años ${ }^{2}$ y sigue siendo el patrón de comparación de cualquier nuevo medicamento antiartrítico, pero también hay que destacar, que los anti TNF- $\alpha$ son eficaces y muchas veces cambia dramáticamente la vida de los enfermos, cuando el metotrexate ha fracasado.

Los anticuerpos monoclonales anti-TNF- $\alpha$ han abierto una nueva era en el tratamiento de algunas enfermedades inflamatorias mediadas inmunológicamente como la $\mathrm{AR}$, la EAA y la enfermedad de Crohn a las que se han agregado psoriasis, síndrome de Behçet, uveítis y otras. Estas enfermedades presentan una vía inflamatoria común en la que el TNF- $\alpha$ juega un papel crucial $^{19}$.

El futuro de los pacientes con artritis inflamatoria inmunológica crónica puede estar mejorando con la introducción de los agentes biológicos. Siguen apareciendo nuevas indicaciones de uso para estas drogas, pero se debe estar atento a la presencia de efectos colaterales o de infecciones por gérmenes oportunistas, las que se han comunicado en el período posterior a su salida al mercado.

sus placebo in rheumatoid arthritis patients receiving concomitant methotrexate. Lancet 1999; 354: 1932-9.

6. Chaudhari U, Romano P, Mulcahy LD, Dooley L, BAKER DG, GotTLEB AB. Efficacy and safety of infliximab monotherapy for plaque-type psoriasis: a randomized trial. Lancet 2001; 357: 1842-7.

7. Braun J, Brandt J, Listing J, ZinK A, Alten W, Golder W ET AL. Treatment of active ankylosing spondylitis with infliximab: a randomized controlled multicentre trial. Lancet 2002; 359: 1187-93.

8. Felson DT, Anderson JJ, Boers M, Bombardier C, Furst D, Goldsmith C et al. American College of Rheumatology. Preliminary definition of improvement in rheumatoid arthritis. Arthritis Rheum 1995; 38: 727-35.

9. Lupsky P, van der Hejde D, St Clair E et al. Infliximab and metothrexate in the treatment of rheumatoid arthritis. N Engl J Med 2000; 343: 1584-602. 
10. Arnett FC, Edworthy SM, Bloch DA, McShane DJ, FRIES JF, CoOPER NS et AL. The American Rheumatism Association 1987 revised criteria for the classification of rheumatoid arthritis. Arthritis Rheum 1988; 31: 315-24.

11. Dougados M, van der Linden S, Juhun R. The European spondyloarthropathy study group preliminary criteria for the classification of spondyloarthropathy. Arthritis Rheum 1991; 34: 1218-27.

12. Pincus T, Calahanan LF, Brooks RH, Fuchs HA, Olsen NJ, KAYE JJ. Self-Report Questionnaire scores in rheumatoid arthritis compared with traditional physical radiographic, and laboratory measures. Ann Intern Med 1989; 110: 259-66.

13. Guidelines for the management of meumatoid arthntis: 2002 Update. Arthritis Rheum 2002; 46: 328-46.

14. Weinberger M, Tierney W, Cowper P, Katz B, BOOHER P. Cost-effectiveness of increased telephone contact for patients with osteoarthritis. A randomized controlled trial. Arthritis Rheum 1993; 36: 243-6.

15. Ware JE JR, Sherbourne CD. The MOS 36-item short-form health survey (SF-36). I. Conceptual framework and item selection. Med Care 1992; 30: 473-83.
16. Keane J, Gershon S, Wise RP, Mirabile-Levens E, Kasznica J, Schwieterman WD et aL. Tuberculosis Associated with Infliximab, a Tumor Necrosis Factor-Neutralizing Agent. N Engl J Med 2001; 345: 1098-104.

17. Mohan N, Edwrds E, Cupps T, Olvverio P, Sandberg G, Crayton H. Demyelination occurring during anti-tumor necrosis alfa therapy for inflammatory arthritides. Arthritis Rheum 2001; 44: 2862-9.

18. Cisternas M, GutiérReZ M, Jacobe山 S. Successful rechallenge with anti-tumor necrosis factor alfa for psoriatic arthritis after development of demyelinating nervous system disease during initial treatment: comment on the article by Mohan et al. Arthritis Rheum 2002; 46: 3107.

19. Braun J, Sieper J. Overview of the use of the antiTNF agent infliximab in chronic inflammatory diseases. Expert Opin Biol Ther 2003; 3: 141-68.

20. Maddison PJ. Is it SLE? Best Pract Res Clin Rheumatol 2002; 16: 167-80.

21. Brown L, Greene M, Gershon S, Edwards ET, Braun M. Tumor necrosis factor antagonist therapy and lymphoma development: Twenty-six cases reported to the Food and Drug Administration. Arthritis Rheum 2002; 46: 3151-8.

Agradecimientos

Los autores agradecen al Dr. Rolando Pastén y a la Srta. EU Marisol Collinao por su atención a los enfermos, a la Srta. MSc Alessandra Gedarlinni por el análisis estadístico de los datos y al Laboratorio Schering-Plough por proporcionar infliximab (Remi$\operatorname{cade}^{\circledR}$ ). 